\title{
Integration of Fresnel Zone Plates in the Bulk of Sapphire Crystal by Femtosecond Laser Pulses
}

\author{
Audrius PUIŠYS, Domas PAIPULAS \\ Laser Research Center, Department of Quantum Electronics, Physics Faculty, Vilnius University, \\ Sauletekio Ave. 10, LT-10223 Vilnius, Lithuania \\ E-mail: domas.paipulas@ff.vu.lt
}

\begin{abstract}
The goal of this study was to integrate Fresnel zone plates with diameters up to $300 \mu \mathrm{m}$ in the bulk of sapphire crystal by direct laser writing technique. These zone plates are intended to be integrated directly into GaN LED substrate because of their potential to increase the light extraction efficiency of UV LED devices. For integration task a femtosecond (320 fs) high repetition rate $(100 \mathrm{kHz}) \mathrm{Yb}: \mathrm{KGW}$ laser system was used together with high precision linear positioning stages. By exposing sapphire to the focused femtosecond laser radiation, regions of modified refractive index were induced and required zone plates were patterned. The discussions about optimal fabrication parameters as well as focusing properties of fabricated zone plates are presented. It is shown that sapphire can be a suitable material for microphotonic device integration: working zone plates with diameters up to $300 \mu \mathrm{m}$ and diffraction efficiencies up to $23 \%$ were integrated below $(80 \mu \mathrm{m})$ the sapphire surface.
\end{abstract}

DOI: $10.2961 /$ jlmn.2015.01.0017

Keywords: Fresnel zone plate, sapphire, femtosecond laser micromachining, direct laser writing

\section{Introduction}

Sapphire due to its excellent mechanical and optical properties is widely used in optics, optoelectronic and microelectronic applications. Besides its wide use in high power laser optics, sapphire gained interest as a good substrate for growing gallium nitride $(\mathrm{GaN})$ layers in light emitting diode (LED) technologies [1]. Unfortunately, because of its hardness and chemical stability, it is difficult to apply traditional machining methods and tools to sapphire crystal, especially when precise machining is needed. However, ultrafast lasers become a promising tool for micromachining all sorts of materials, including sapphire. Cutting and micropatterning of sapphire surface is extensively studied nowadays [2]. Moreover, with femtosecond laser machining it is possible to integrate various photonic devices directly into the bulk of transparent materials [3, 4]. This technology was successfully demonstrated in a wide variety of glasses; however, the integration capabilities into sapphire are not well documented. It is known that focused femtosecond laser pulses can modify sapphire properties [5], but this result is still waiting to be exploited.

Most of the reported studies, related to sapphire, focus on the research of fundamental light-mater interaction. These are the studies on the laser induced damage threshold [6], surface ablation [7, 8], surface and in-bulk structuring and crystal amorphisation $[9,10]$, etc. However, there are almost no reports on the integration capabilities of various photonic devices, such as a Fresnel zone plates, inside sapphire. Moreover, most of the reported studies on the fabrication of these plates, use fused silica [11-15], whereas studies on the fabrication capabilities inside sapphire, to our knowledge, haven't been reported.

In this work we attempted to integrate micro Fresnel zone plates with diameters up to $300 \mu \mathrm{m}$ in the volume of sapphire crystal by direct laser writing technique. By exposing sapphire to focused femtosecond laser radiation, regions of modified refractive index were induced and required Fresnel zone plates were patterned.

Fresnel plate consists of series of concentric discs (Fresnel zones) which periodically alter light transmittance properties. Boundaries of each disk can be approximated by radii which are $r_{n} \approx \sqrt{n \lambda f}$, where the integer $n$ indicates the $n^{\text {th }}$ Fresnel zone, $\lambda$ is the operating wavelength and $f$ is the focal length [12]. If odd or even Fresnel zones are blocked or induce sufficient phase change in transmitted light, such zone plate has focusing property. The working principle of a zone plate relies on the diffraction of light, $i$. e. the incident monochromatic light is diffracted by the periodically alternating zones and, depending on the dimensions of the zones, constructively interferes at a designed focal point. Therefore, with direct laser writing it is possible to selectively modify material optical properties, by producing concentric disks with diameters $r_{2}-r_{1}, r_{4}-r_{3}$, $\ldots, r_{2 \mathrm{n}}-r_{2 \mathrm{n}-1}, \ldots$ thus forming in the bulk integrated Fresnel zone plate. Theoretical resolution of such plate is expressed as $\delta=1.22 \times \Delta r_{\max }$, where $\Delta r_{\max }$ is plate's outermost disk thickness.

These zone plates are intended to be integrated directly into GaN LED substrate: due to the fact that sapphire can transmit UV wavelengths, the ability of a zone plate to focus this type of radiation and the fact that the largest volume of sapphire production is expected to be for substrates for high brightness LED devices [16], it would be advantageous to employ sapphire not only as a substrate but also as a medium for integration of zone plates which could potentially increase the light extraction efficiency of UV LED devices. 


\section{Experimental setup}

For integration tasks we used an $\mathrm{Yb}: \mathrm{KGW}$ based "Pharos" laser system ("Light Conversion Ltd.") as a femtosecond laser source. This laser produces high intensity $320 \mathrm{fs}$ duration pulses with $1030 \mathrm{~nm}$ wavelength. Laser pulse repetition rates up to $200 \mathrm{kHz}$ were used. The setup of the experiment is shown in Fig. 1. Laser pulses are first attenuated and then focused into the bulk of sapphire wafer with a 0.6-NA (numerical aperture) objective lens. The sapphire sample was mounted on a 3-axis nanopositioning system based on the "Aerotech ANT180" linear motion stages having accuracy of $150 \mathrm{~nm}$. By translating the sample perpendicular to the laser beam in a spiral trajectory, regions of modified refractive index were induced and required zone plates were patterned.

The focusing properties of the manufactured zone plates were investigated with a $\mathrm{He}-\mathrm{Ne}$ laser having $0.8 \mathrm{~mW}$ average power and $633 \mathrm{~nm}$ wavelength; also white light LED source was used for inspection. The testing setup for the focusing properties of the manufactured zone plates is shown in Fig. 2. Before hitting the sapphire sample, the diameter of the $\mathrm{He}$ - Ne laser beam was expanded in order to increase illumination homogeneity on the sample. The intensity distribution of the diffracted beam was investigated by moving an imagining system consisting of a 10x objective, a tube lens and a CCD camera along the direction of the laser beam propagation.

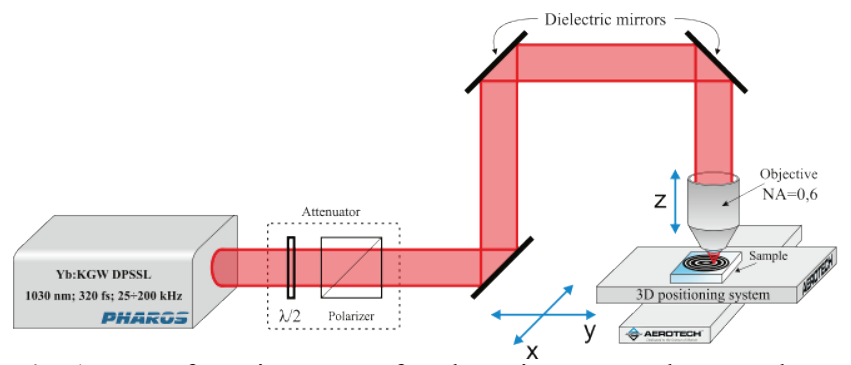

Fig. 1. Manufacturing setup for the micro Fresnel zone plates: femtosecond laser and 3 axis sample positioning system.

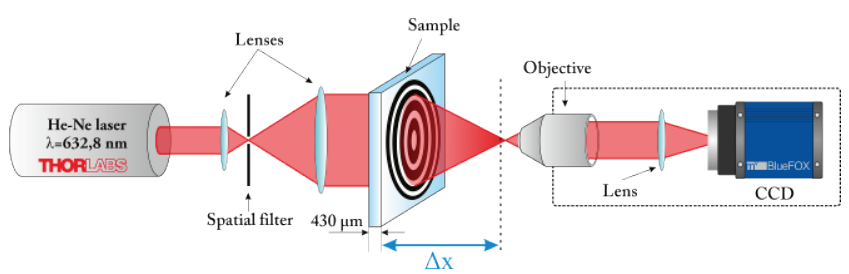

Fig. 2. Testing setup for the focusing properties of the zone plates, integrated in the bulk of sapphire wafer.

\section{Results}

Writing pulse energy, pulse repetition rate and translation speed of the sample play major roles in creating uniformly-modified sapphire regions without obvious macroscopic damage. At first a series of plane diffractive gratings were recorded with different laser pulse parameters (pulse energy and laser repetition rate) in the bulk of sapphire in order to investigate general behavior of laser induced micromodifications (Fig. 3.). Gratings dimensions were $200 \mu \mathrm{m} \times 200 \mu \mathrm{m}$ and each had $4 \mu \mathrm{m}$ period. A fixed sam- ple translation speed value of $300 \mu \mathrm{m} / \mathrm{s}$ was used to ensure that the manufacturing time of a single diffraction grating wouldn't exceed $90 \mathrm{~s}$.

The outcomes of the manufacturing process of diffraction gratings were inspected under the optical microscope in phase contrast regime. It was determined that across all tested pulse repetition rate range $(25 \mathrm{kHz}-200 \mathrm{kHz})$, laser pulse energy of $300 \mathrm{~nJ}$ (corresponding to $17 \mathrm{~J} / \mathrm{cm}^{2}$ energy fluence and $5 \times 10^{13} \mathrm{~W} / \mathrm{cm}^{2}$ peak intensity) was sufficient to form homogenous modified regions of sapphire without any visual presence of microcracks or microfractures inside the sapphire crystal. With lower pulse energies modification tend to be inhomogeneous, while increasing the value up to $900 \mathrm{~nJ}$ and beyond lead to the formation of macroscopic fractures. These results show that at tested laser repetition rate range accumulation effects do not play major role in modification creation. Therefore, we used pulse repetition rate of $100 \mathrm{kHz}$ as an optimal value.

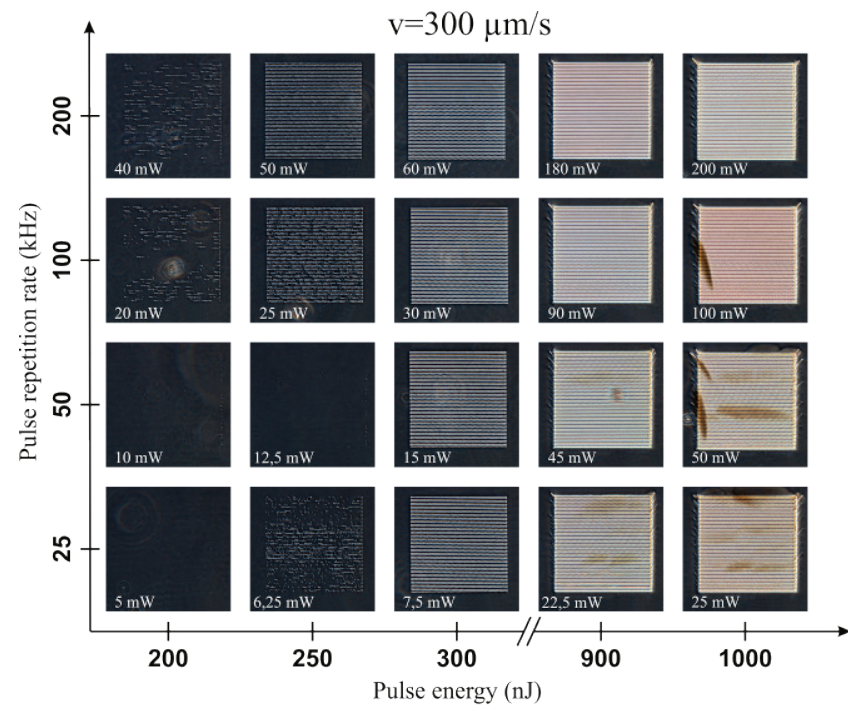

Fig. 3. The impact of the laser pulse energy and pulse repetition rate on the visual uniformity and evenness of diffraction gratings integrated in the bulk of sapphire. The dimensions of $200 \times 200 \mu \mathrm{m}$ and a period of $4 \mu \mathrm{m}$ for each diffraction grating were chosen. Phase contrast microscopy was used. The brightness of each photographed diffraction grating differs due to the fact that for each grating different automatic exposure and white balance values of the CCD were used.

Several layers of diffraction gratings on top of each other were also manufactured in order to increase the overall axial thickness of modified region in the sapphire crystal. A typical axial length of a single layer grating, recorded with $300 \mathrm{~nJ}$ pulses and 0.6-NA objective lens is approximately $12 \mu \mathrm{m}$ (Fig. 4). Stitching several layers of gratings is possible in the sapphire, however axial shift of the layers has to be slightly larger, otherwise stress induced microcracks develops in the crystal. This prevents formation of continuously long modified regions in axial dimensions. In Fig. 4 b) it is own a crack-free stitching of three layers with separation of $14 \mu \mathrm{m}$ between layers. It is known, that the stacking of layers can highly increase the diffraction efficiency of simple plane grating [17] as well as more complex Fresnel plate [18]. 

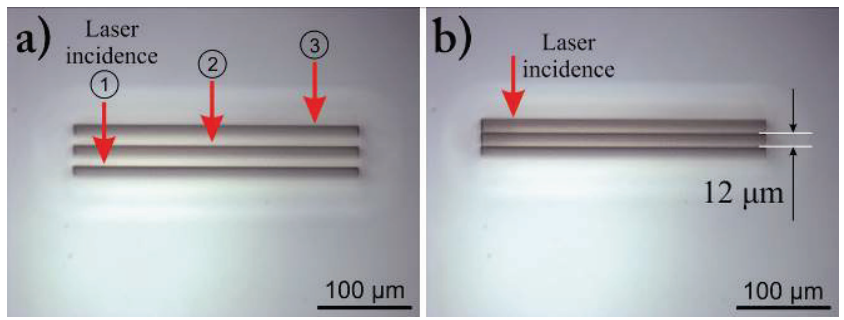

Fig. 4. Profile of 3 layers of diffraction gratings fabricated in the bulk of sapphire. Separation between the center of the layers is $20 \mu \mathrm{m}$ (a) and $14 \mu \mathrm{m}$ (b). It was determined that the axial length of a modified region of sapphire for 300 $\mathrm{nJ}$ pulses focused with a 0.6 -NA lens is approximately 12 $\mu \mathrm{m}$.

Similar tendencies of the visual quality of the manufactured photonic devices inside the bulk of sapphire are present when Fresnel zone plates are fabricated. A $300 \mathrm{~nJ}$ pulse energy ensured the production of crack-free zone plates, while higher energies can lead to formation of micro and macro cracks as can be seen in Fig. 5 .

Sample translation speed also plays an important role in recorded plate quality. When circular geometry object is recorded with linear motion stages, tables have to be moved in polygon pattern which includes many acceleration/deceleration steps, leading to the developing of table oscillations. Also in our setup, the inertia of the tables in orthogonal directions was not the same (one table is mounted on the other), thus at higher translation velocities positioning accuracy, especially in one direction, worsens when circular shape is drawn. This effect is indicated in Fig. 6 . The maximum translation speed at which curved homogeneous microstructures were successfully recorded were limited by the radius of the curved structure itself: i.e. by decreasing the diameter of a zone plate from $260 \mu \mathrm{m}$ to 150 $\mu \mathrm{m}$ the threshold value of the translation velocity at which microcracks started to form decreased from $600 \mu \mathrm{m} / \mathrm{s}$ to $400 \mu \mathrm{m} / \mathrm{s}$. Also stress-induced microcracks start to appear along one diagonal of Fresnel plates as indicated in Fig. 6 $\mathrm{b}, \mathrm{d})$. Most prominent fracturing is observed at outer rings which, we suppose, are caused by table oscillation resonance. When circle radius increases, the distance between polygon edges also increase which, given the constant linear speed, decreases acceleration/deceleration rate. At particular writing conditions this rate can resonate with stage self-oscillations highly amplifying them and reducing table accuracy. Reduced accuracy increases additional pulse overlap that results in sample overexposure and material damage. This effect prevents the recording of Fresnel plates with high velocity using linear stages.
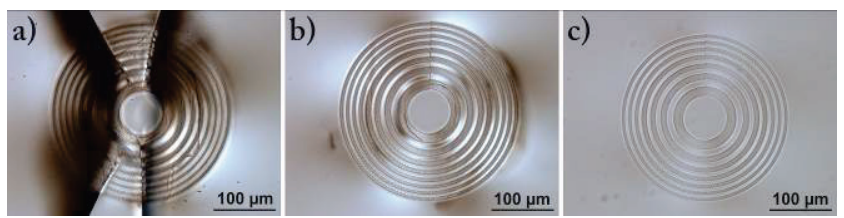

Fig. 5. Possible outcomes of the manufacturing process: a) macrocracks $(1.2 \mu \mathrm{J}, 50 \mathrm{kHz}, 200 \mu \mathrm{m} / \mathrm{s}) ; \mathrm{b})$ microcracks $(1 \mu \mathrm{J}$, $50 \mathrm{kHz}, 200 \mu \mathrm{m} / \mathrm{s}) ; \mathrm{c})$ a crack-free zone plate (300 nJ, 100 $\mathrm{kHz}, 200 \mu \mathrm{m} / \mathrm{s})$.
Several layers of Fresnel zone plates stacked on top of each other were also formed in the bulk of sapphire crystal. Despite the fact, that according to Fig. 4, manufactured plane diffraction gratings didn't manifest any visual signs of microcrack formation with layer separation of $14 \mu \mathrm{m}$, this result didn't repeat itself when the same values of layer separation was used for multi-layer Fresnel zone plate fabrication (all manufactured multilevel zone plates possessed distinctive macrocracks that could be seen with a naked eye (Fig. 7 a)). Successful fabrication of multilayered Fresnel plates was possible when distance between the layers was increased to $50 \mu \mathrm{m}$ (Fig. $7 \mathrm{~b}$ ). This effect shows that curved microstructures are more prone in inducing stress in the sapphire.
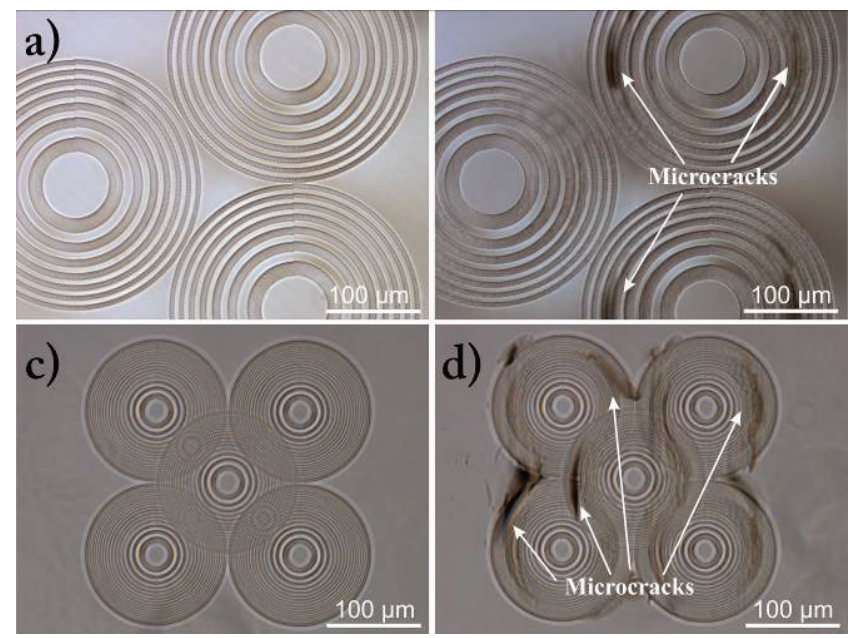

Fig. 6. Searching for the optimal value of sample scanning speed while retaining $300 \mathrm{~nJ}$ pulse energy and $100 \mathrm{kHz}$ pulse repetition rate. Zone plates having diameter of $260 \mu \mathrm{m}$ were recorded with a) $200 \mu \mathrm{m} / \mathrm{s}$ and b) $600 \mu \mathrm{m} / \mathrm{s}$ translation velocity. Zone plates with $150 \mu \mathrm{m}$ diameter were recorded with c) $200 \mu \mathrm{m} / \mathrm{s}$ and d) $400 \mu \mathrm{m} / \mathrm{s}$ velocity.

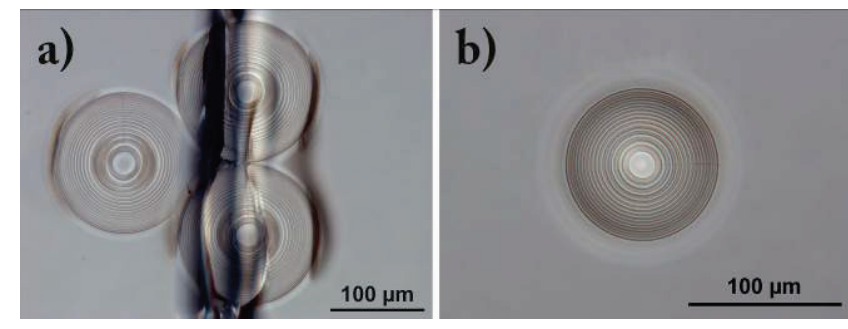

Fig. 7. Multilayer Fresnel zone plates inside the bulk of sapphire: a) 3 plates consisting of 3 layers stacked $10 \mu \mathrm{m}$ apart (top view); b) single plate consisting of 3 layers $30 \mu \mathrm{m}$ apart (top view).

Various arrangements of zone plates up to 3 layers and diameters ranging from $150 \mu \mathrm{m}$ to $300 \mu \mathrm{m}$ and focal lengths from $500 \mu \mathrm{m}$ to $3 \mathrm{~mm}$ were formed in sapphire crystal and their focusing properties were investigated. Fresnel plate focusing effect was clearly present when samples were irradiated with collimated white light source as can be seen in Fig. 8. One can see that zone plates show light focusing behavior event when they are interleaved with each other (such plate system is also shown in Fig. 5 c)). By illuminating these plates with a $\mathrm{He}-\mathrm{Ne}$ laser and measuring its intensity at the theoretical focus position (Fig. 
9) and the reference position (surface of plate) light focusing efficiencies were evaluated. Measured efficiencies, and focal spot diameters (@ FWHM) of manufactured zone plates are shown in Fig.10 and Fig.11 respectively. Lowest efficiencies were achieved with zone plates designed to work at small focal distance $(0.5 \mathrm{~mm})$, while for longer focal lengths efficiencies tended to increase reaching values even up to $23 \%$ at multilayer configuration. The outermost Fresnel zone number of $300 \mu \mathrm{m}$-diameter grating was 14 in our experiments, thus for $0.5 \mathrm{~mm}$ focal length plate theoretical resolution was $2.1 \mu \mathrm{m}\left(\Delta r_{14-13}=1.7 \mu \mathrm{m}\right)$. For longer focal lengths the theoretical resolutions are: $\delta_{1 \mathrm{~mm}}=2.9 \mu \mathrm{m} ; \delta_{2 \mathrm{~mm}}=4.1 \mu \mathrm{m}, \delta_{3 \mathrm{~mm}}=5.1 \mu \mathrm{m}$. Measured resolutions were slightly larger, but comparable to theoretic value and have correlation with efficiency data.

\section{Discussion}

For infinitely large ideal amplitude-type zone plate maximum theoretical diffraction efficiencies do not exceed $10.1 \%$ [19]. Relatively high focusing efficiencies achieved in our experiment show that zone plates integrated in sapphire is of phase-type. This conclusion is supported by a fact, that efficiency can be increased with multilayering strategy. The buildup of phase shift can be attributed to the change of refractive index at laser affected areas. It is known that crystal amorphization and void-like structures with sharp decrease in refractive index can develop under intense femtosecond irradiation [20], also the appearance of stress-induced birefringence near modified regions tend to increase refractive index which rises overall refractive index modulation [21].

The smallest feature recorded in bulk sapphire with 0.6NA objective was $\sim 1 \mu \mathrm{m}$ in diameter, and this limits recoding of higher order Fresnel zones, also such resolution prevents formation of high-NA Fresnel zone plates because in this case submicrometric modification control is required. This explains low diffraction efficiencies for short focal length plates, and multilayering do not add any benefit to plate performance. When distances between Fresnel zone plates becomes greater (plates with longer focal lengths) multilayering can boost the performance, however there exist optimal stacking parameters (number of layers, overlapping distance) that show best performance. As layer stacking cannot be homogeneous, appearing axial modulation of refractive index and unknown actual modification amplitude complicates theoretical evaluation of most optimal Fresnel zone plates.
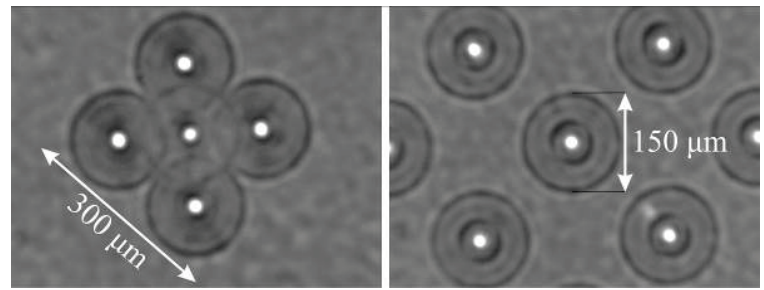

Fig. 8. Light focusing effect of various arrangements of zone plates integrated into the bulk of sapphire with collimated white light (LED). Image was taken by imaging the focal position of Fresnel plate $(0.4 \mathrm{~m})$ with microscope objective on CCD camera.
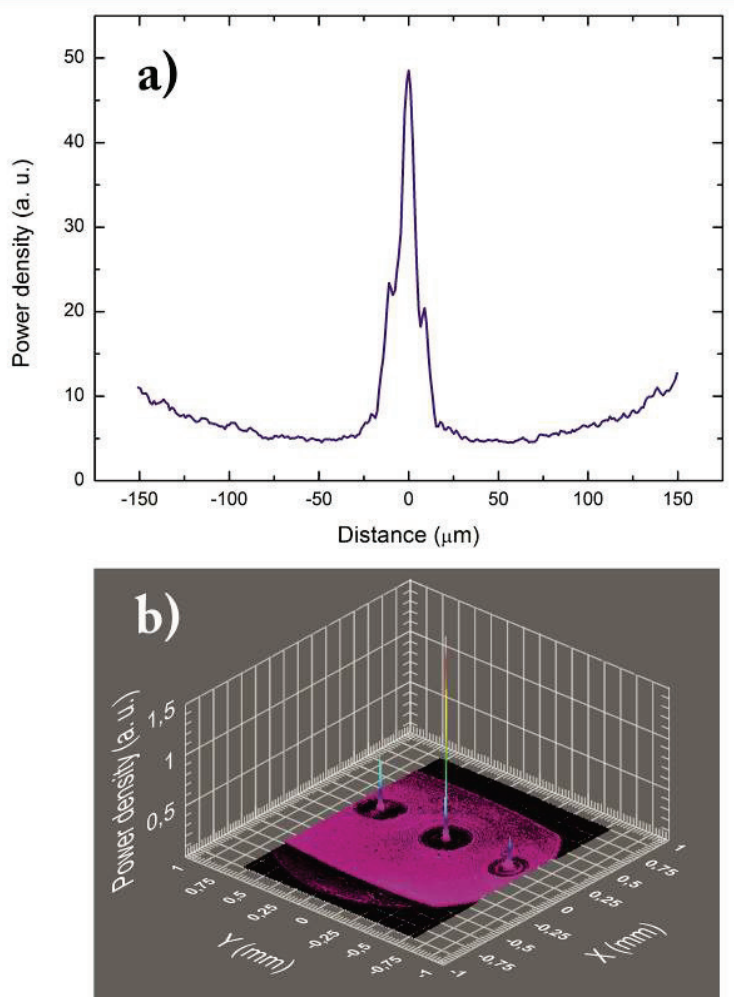

Fig. 9. One-dimensional (a) and two-dimensional (b) light intensity distribution at the focal plane of a 2-layer Fresnel zone plate inside the bulk of sapphire. The layers of the zone plate are $50 \mu \mathrm{m}$ apart. The zone plates diameter are $300 \mu \mathrm{m}$, the focal length is $2 \mathrm{~mm}$.

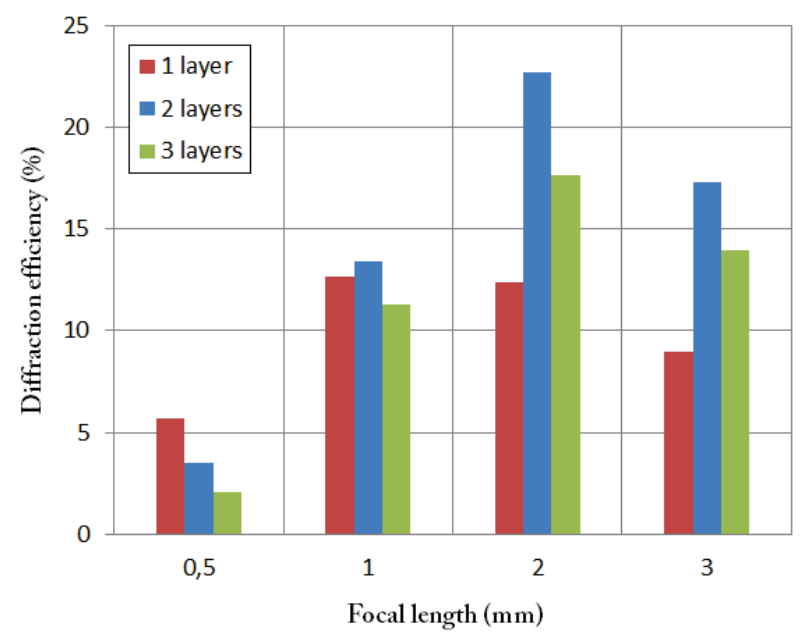

Fig. 10. Focusing efficiencies of Fresnel zone plates having different focal length and number of layers. All plates had diameter of $300 \mu \mathrm{m}$. 


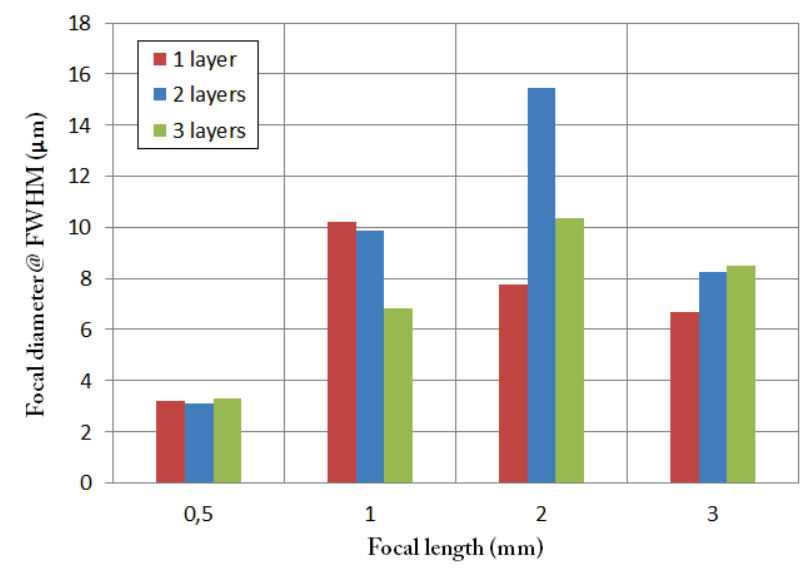

Fig. 11. The focused spot size at the theoretical focal plane of manufactured plates. All plates had diameter of $300 \mu \mathrm{m}$.

\section{Conclusions}

In summary, it was demonstrated that sapphire can be a suitable material for microphotonic device integration. By exposing sapphire to focused femtosecond laser radiation, working zone plates with diameters up to $300 \mu \mathrm{m}$ were integrated below $(80 \mu \mathrm{m})$ the sapphire surface. By employing optimal laser radiation and fabrication parameters (300 $\mathrm{nJ}$ pulse energy, $100 \mathrm{kHz}$ repetition rate and translation speed up to $400 \mu \mathrm{m} / \mathrm{s}$ ) patterned zone plates showed no visual sign of cracks or micro fractures inside the sapphire crystal even when they overlapped. Quick testing reveals the fact that manufactured zone plates exhibit light focusing properties as expected. Diffraction efficiencies up to $23 \%$ were reached. However, closer inspection of these focusing properties and optimization of the manufacturing process is still needed in order to apply this technology for sapphire substrates used in LED manufacturing.

\section{Acknowledgment}

Authors acknowledge financial support by a research grant No. VP1-3.1-ŠMM-10-V-02-007 (Development and Utilization of a New Generation Industrial Laser Material Processing Using Ultrashort Pulse Lasers for Industrial Applications) from the European Social Fund Agency. We thank R. Tomašiūnas, A. Kadys, I. Reklaitis, E. Jelmakas, and S. Juodkazis for valuable discussions on this subject.

\section{References}

[1] E. R. Dobrovinskaya, L. A. Lytvynov and V. Pishchik: "Sapphire: Material, Manufacturing, Applications", (Springer, New York, 2009) p. 481.

[2] C.-W. Chang, C.-Y. Chen, T.-L. Chang, C.-J. Ting, C.-P. Wang, and C.-P. Chou: Appl. Phys. A, 109, (2012) 441.

[3] K. M. Davis, K. Miura, N. Sugimoto, and K. Hirao: Opt. Lett., 21, (1996) 1729.
[4] G. D. Valle, R. Osellame and P. Laporta: J. Opt. A. Pure Appl. Opt., 11, (2009) 013001.

[5] S. Juodkazis, K. Nishimura, H. Misawa, T. Ebisui, R. Waki, S. Matsuo, and T. Okada: Adv. Mater., 18, (2006) 1361 .

[6] O. Uteza, B. Bussière, F. Canova, J.-P. Chambaret, P. Delaporte, T. Itina and M. Sentis: Appl. Surf. Sci., 254, (2007) 799.

[7] X. C. Wang, G. C. Lim, H. Y. Zheng, F. L. Ng, W. Liu and S. J. Chua: Appl. Surf. Sci., 228, (2004) 221.

[8] L. Qi, K. Nishii, M. Yasui, H. Aoki and Y. Namba: Opt. Lasers Eng., 48, (2010) 1000.

[9] S. Juodkazis, K. Nishimura and H. Misawa: Appl. Surf. Sci., 253, (2007) 6539.

[10] V. Mizeikis, S. Kimura, N. V. Surovtsev, V. Jarutis, A. Saito, H. Misawa and S. Juodkazis: Appl. Surf. Sci., 255, (2009) 9745.

[11] W. Watanabe, D. Kuroda, K. Itoh, and J. Nishii: Opt. Express, 10, (2002) 978.

[12] P. Srisungsitthisunti, O. K. Ersoy and X. Xu: J. Opt. Soc. Am. B, 24, (2007) 2090.

[13] I.-B. Sohn, M. S. Ahsan, Y.-C. Noh, H.-K. Choi, J.-T. Kim and M. J. Ko: Proc. SPIE, 8923, (2013) 89233Y.

[14] P. Srisungsitthisunti, M. Campus, O. K. Ersoy and X. Xu: J. Opt. Soc. Am. A, 26, (2009) 2114.

[15] E. Bricchi, J. D. Mills, P. G. Kazansky, B. G. Klappauf and J. J. Baumberg: Opt. Lett., 27, (2002) 2200.

[16] M. S. Akselrod and F. J. Bruni: J. Cryst. Growth, 360, (2012) 134.

[17] T. Toma, Y. Furuya, W. Watanabe, K. Itoh, J. Nishii, and K. Hayashi: Opt. Rev., 7, (2000) 14.

[18] P. Srisungsitthisunti, O. K. Ersoy and X. Xu: Appl. Phys. Lett., 90, (2007) 011104.

[19] K. Yamada, W. Watanabe, Y. Li, K. Itoh, and J. Nishii: Opt. Lett., 29, (2004) 1846.

[20] S. Juodkazis, K. Nishimura, S. Tanaka, H. Misawa, E. G. Gamaly, B. Luther-Davies, L. Hallo, P. Nicolai, and V. T. Tikhonchuk: Phys. Rev. Lett., 96, (2006) 166101 .

[21] J. Morikawa, A. Orie, T. Hashimoto, S. Juodkazis: Opt. Express, 18, (2010) 8300.

(Received: June 30, 2014, Accepted: January 21, 2015) 